\title{
PROJECTIONS IN DUALS TO ASPLUND SPACES MADE WITHOUT SIMONS' LEMMA
}

\author{
MAREK CÚTH AND MARIÁN FABIAN \\ In honor of the 70th birthday of Charles Stegall.
}

\begin{abstract}
G. Godefroy and the second author of this note proved in 1988 that in duals to Asplund spaces there always exists a projectional resolution of the identity. A few years later, Ch. Stegall succeeded to drop from the original proof a deep lemma of S. Simons. Here, we rewrite the condensed argument of $\mathrm{Ch}$. Stegall in a more transparent and detailed way. We actually show that this technology of Ch. Stegall leads to a bit stronger/richer object - the so called projectional skeleton - recently constructed by W. Kubiś, via S. Simons' lemma and with help of elementary submodels from logic.
\end{abstract}

In 1988, G. Godefroy and the second named author of this note constructed in [FG] a projectional resolution of the identity in duals to general Asplund spaces; see, e.g., [F, Definitions 1.0.1 and 6.1.5]. A few years later, Ch. Stegall, presented a simplified variant of this construction by avoiding from the proof the use of S. Simons' lemma [F, Lemma 8.1.3] or of any other substitute of it. He published it, in a rather condensed form in [St]. In this note, we perform his argument in full details. Thus we believe that Ch. Stegall's approach, so far overlooked by audience, will attract a broader attention. Ch. Stegall's technology led us even to constructing a stronger/richer object, which includes the so called 1-projectional skeleton, introduced and studied recently by W. Kubiś, see [K, pages 765, 766], [KKL, pages 369, 370]. W. Kubiś' construction was based on S. Simons' lemma and was done by using elementary submodels from logic.

Let $(X,\|\cdot\|)$ be any Banach space. If $V$ is a subspace of $X$ and $x^{*} \in X^{*}$, then $x^{*} \uparrow_{V}$ means the restriction of $x^{*}$ to $V$; similarly, we put $M \uparrow_{V}:=\left\{\left.x^{*}\right|_{V}: x^{*} \in M\right\}$ for any set $M$ in $X^{*}$. Alaoglu's theorem asserts that the closed unit ball $B_{X^{*}}$ in $X^{*}$ provided with the weak* topology is a compact space. Let $\mathcal{C}\left(B_{X^{*}}\right)$ denote the (Banach) space of all continuous functions on this compact space, endowed

Date: June 19, 2018.

2010 Mathematics Subject Classification. Primary 46B26, Secondary 46B20, 46B22.

Key words and phrases. Asplund space, projectional resolution of identity, projectional skeleton, weak star dentability, Jayne-Rogers selection theorem, separable reduction.

M. Cúth was supported by the Grant No. 282511/B-MAT/MFF of Grant Agency of Charles University in Prague. M. Fabian was supported by grant P201/12/0290 and by RVO: 67985840. 
with the maximum norm $\|\cdot\|$. Consider the multivalued mapping

$$
\mathcal{C}\left(B_{X^{*}}\right) \ni f \longmapsto\left\{x^{*} \in B_{X^{*}}: f\left(x^{*}\right)=\max f\left(B_{X^{*}}\right)\right\}=: \partial(f) \subset B_{X^{*}} ;
$$

thus $\partial: \mathcal{C}\left(B_{X^{*}}\right) \longrightarrow 2^{X^{*}}$. Clearly, for every $f \in \mathcal{C}\left(B_{X^{*}}\right)$ the set $\partial(f)$ is nonempty and weak* compact. The mapping $\partial$ is also norm-to-weak* upper semicontinuous. To check this, consider any weak* open set $W$ in $X^{*}$. We have to show that $\left\{f \in \mathcal{C}\left(B_{X^{*}}\right): \partial(f) \subset W\right\}$ is an open set. Assume that this is not the case. Then there exists a sequence $f_{0}, f_{1}, f_{2}, \ldots$ of elements in $\mathcal{C}\left(B_{X^{*}}\right)$ such that $\partial\left(f_{0}\right) \subset W$, that $\left\|f_{n}-f_{0}\right\| \rightarrow 0$ as $n \rightarrow \infty$, and that for every $n \in \mathbb{N}$ there is $x_{n}^{*} \in \partial\left(f_{n}\right) \backslash W$. Recalling that all the $x_{n}^{*}$ 's belong to the (weak* compact) set $B_{X^{*}}$, the sequence $\left(x_{n}^{*}\right)$ has a weak* cluster point, $x^{*} \in B_{X^{*}}$, say. Also

$$
\begin{aligned}
\max f_{0}\left(B_{X^{*}}\right) & \leq \max f_{n}\left(B_{X^{*}}\right)+\left\|f_{0}-f_{n}\right\| \\
& =f_{n}\left(x_{n}^{*}\right)-f_{0}\left(x_{n}^{*}\right)+\left\|f_{0}-f_{n}\right\|+f_{0}\left(x_{n}^{*}\right) \leq 2\left\|f_{n}-f_{0}\right\|+f_{0}\left(x_{n}^{*}\right)
\end{aligned}
$$

for every $n \in \mathbb{N}$. And, since $f_{0}$ is weak* continuous, $\left(f_{0}\left(x^{*}\right) \leq\right) \max f_{0}\left(B_{X^{*}}\right) \leq$ $f_{0}\left(x^{*}\right)$. We got that $x^{*} \in \partial\left(f_{0}\right)(\subset W)$. However, simultaneously, $x^{*} \notin W$ as $x_{n}^{*} \notin W$ for all $n \in \mathbb{N}$; a contradiction.

If the Banach space $(X,\|\cdot\|)$ is separable, the mapping $\partial: \mathcal{C}\left(B_{X^{*}}\right) \longrightarrow 2^{X^{*}}$ has the following (crucial) property: For every $\xi \in B_{X^{*}}$ there is an $f \in \mathcal{C}\left(B_{X^{*}}\right)$ such that $\partial(f)=\{\xi\}$. (This is the very point where the approach of Ch. Stegall starts.) Indeed, since the compact space $\left(B_{X^{*}}, w^{*}\right)$ is then metrizable, $\xi$ is a $G_{\delta}$ point in it and Urysohn's lemma [E, Theorem 1.5.10] provides easily a suitable function $f$. More specifically (and without using any topological tool), such an $f$ can be constructed by the formula

$$
B_{X^{*}} \ni x^{*} \longmapsto 1-\sum_{n=1}^{\infty} 2^{-n}\left|\left\langle\xi, s_{n}\right\rangle-\left\langle x^{*}, s_{n}\right\rangle\right|=: f\left(x^{*}\right)
$$

where $\left\{s_{1}, s_{2}, \ldots\right\}$ is a fixed countable dense subset of the closed unit ball $B_{X}$ in $X$. (By [F, Lemma 2.2.1(ii)] we know that the function $C\left(B_{X^{*}}\right) \ni g \longmapsto$ $\max g\left(B_{X^{*}}\right)$ is Gateaux differentiable at $g:=f$.)

In what follows, let $(X,\|\cdot\|)$ be a Banach space of an arbitrary density. The observation in the latter paragraph leads to introducing the following family of functions from $\mathcal{C}\left(B_{X^{*}}\right)$. Let $S$ be any nonempty set in $B_{X}$. By $\mathcal{L}(S)$ we denote the family of all functions of the form

$$
B_{X^{*}} \ni x^{*} \longmapsto 1-\sum_{n=1}^{k} 2^{-n}\left|a_{n}-\left\langle x^{*}, s_{n}\right\rangle\right|,
$$

where $k \in \mathbb{N}, a_{1}, a_{2}, \ldots, a_{k}$ are rational numbers in $[-1,1]$, and $s_{1}, s_{2}, \ldots, s_{k}$ are elements from $S$; note that $\# \mathcal{L}(S)=\# S+\aleph_{0}$. It is easy to check that each element of $\mathcal{L}(S)$ is weak* continuous and has maximum norm at most equal to 1. Thus $\mathcal{L}(S)$ is a subset of (the closed unit ball of) $\mathcal{C}\left(B_{X^{*}}\right)$. We can easily 
check that

$$
\overline{\mathcal{L}(\bar{S})}=\overline{\mathcal{L}(S)}
$$

hence, the set in (2) is separable whenever $S$ is separable. Note that the $f$ defined in (11) belongs to the (norm) closure of $\mathcal{L}\left(\left\{s_{1}, s_{2}, \ldots\right\}\right)$.

From now on, assume that $(X,\|\cdot\|)$ is an Asplund space, which (equivalently) means that the dual space $X^{*}$ is weak* dentable [Ph, Theorem 2.32]. Then, we are ready to apply the selection theorem of Jayne and Rogers [JR, Theorem 8], [F, Theorem 8.1.2] to our multivalued mapping $\partial$ (which is already known to be norm-to-weak* upper semicontinuous and weak* compact valued). Thus we get a sequence $\lambda_{j}: \mathcal{C}\left(B_{X^{*}}\right) \longrightarrow X^{*}, j \in \mathbb{N}$, of norm-to-norm continuous mappings such that for every $f \in \mathcal{C}\left(B_{X^{*}}\right)$ the $\operatorname{limit}_{j \rightarrow \infty} \lambda_{j}(f)=: \lambda_{0}(f)$ exists in the norm topology of $X^{*}$ and moreover $\lambda_{0}(f) \in \partial(f)$, that is, $f\left(\lambda_{0}(f)\right)=$ $\max f\left(B_{X^{*}}\right)$. Now, we define the multivalued mapping

$$
\mathcal{C}\left(B_{X^{*}}\right) \ni f \longmapsto\left\{\lambda_{1}(f), \lambda_{2}(f), \ldots\right\}=: \Lambda(f) \subset X^{*} ;
$$

thus $\Lambda: \mathcal{C}\left(B_{X^{*}}\right) \longrightarrow 2^{X^{*}}$. The continuity of the mappings $\lambda_{j}$ 's and (2) then guarantee that

$$
\overline{\Lambda(\overline{\mathcal{L}(\bar{S})})}=\overline{\Lambda(\mathcal{L}(S))}
$$

Proposition 1. Let $(X,\|\cdot\|)$ be any Asplund space and let $V$ be any subspace of it. Then $B_{V^{*}} \subset \overline{\Lambda\left(\mathcal{L}\left(B_{V}\right)\right)} \Upsilon_{V}$ and dens $V=\operatorname{dens} \overline{\operatorname{sp} \Lambda\left(\mathcal{L}\left(B_{V}\right)\right)}=\operatorname{dens} V^{*}$; in particular, $B_{X^{*}} \subset \overline{\Lambda\left(\mathcal{L}\left(B_{X}\right)\right)}$.

Proof. First, assume that $V$ is separable. Consider any $v^{*} \in B_{V^{*}}$. Find a countable dense subset $\left\{s_{1}, s_{2}, \ldots\right\}$ of $B_{V}$. Define $f:=1-\sum_{n=1}^{\infty} 2^{-n} \mid\left\langle v^{*}, s_{n}\right\rangle-$ $\left\langle\cdot, s_{n}\right\rangle \mid$; thus $f \in \overline{\mathcal{L}\left(B_{V}\right)}$. Then $f\left(x^{*}\right)=1$ for every $x^{*} \in B_{X^{*}}$, with $x^{*} \uparrow_{V}=v^{*}$, and $-1 \leq f\left(y^{*}\right)<1$ whenever $y^{*} \in B_{X^{*}}$ and $y^{*} \uparrow_{V} \neq v^{*}$. Thus $\partial(f) \uparrow_{V}=\left\{v^{*}\right\}$, and, since $\lambda_{0}(f) \in \partial(f)$, we have that $\lambda_{0}(f) \uparrow_{V}=v^{*}$. Recalling that $\| \lambda_{j}(f)-$ $\lambda_{0}(f) \| \longrightarrow 0$ as $j \rightarrow \infty$, we can conclude that

$$
\lambda_{0}(f) \in \overline{\Lambda(f)} \subset \overline{\Lambda\left(\overline{\mathcal{L}\left(B_{V}\right)}\right)}=\overline{\Lambda\left(\mathcal{L}\left(B_{V}\right)\right)}
$$

by (3). Therefore, $v^{*}=\lambda_{0}(f) \uparrow_{V} \in \overline{\Lambda\left(\mathcal{L}\left(B_{V}\right)\right)} \uparrow_{V}$.

Second, assume that $V$ is non-separable (provided that $X$ is non-separable). Consider any $v^{*} \in B_{V^{*}}$. Find $\xi \in B_{X^{*}}$ so that $\xi \uparrow_{V}=v^{*}$. We shall construct countable sets $S_{0} \subset S_{1} \subset S_{2} \subset \cdots \subset B_{V}$ and separable subspaces $Z_{0} \subset Z_{1} \subset$ $Z_{2} \subset \cdots \subset V$ as follows. Pick any countable subset $S_{0}$ of $B_{V}$ and any separable (rather infinite-dimensional) subspace $Z_{0} \subset V$. Let $m \in \mathbb{N}$ be given and assume that we have found $S_{m-1}$ and $Z_{m-1}$. Clearly, $\Lambda\left(\mathcal{L}\left(S_{m-1}\right)\right)$ is a countable subset of $X^{*}$. Find then a countable set $S_{m-1} \subset S_{m} \subset B_{V}$ such that $\overline{S_{m}} \supset B_{X} \cap Z_{m-1}$ 
and that

$$
\left\|\xi-x^{*}\right\|=\sup \left\{\langle\xi, s\rangle-\left\langle x^{*}, s\right\rangle: s \in S_{m}\right\} \text { for every } x^{*} \in \Lambda\left(\mathcal{L}\left(S_{m-1}\right)\right) .
$$

Put then $Z_{m}:=\overline{\operatorname{sp}\left(Z_{m-1} \cup S_{m}\right)}$. Do so for every $m \in \mathbb{N}$ and put finally $S:=$

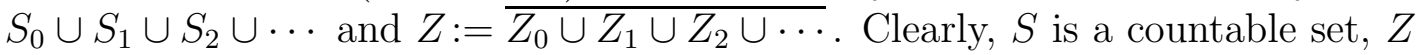
is a separable subspace of $V$, and $\bar{S}=B_{Z}$. The "separable" case says that $\left.\xi\right\rceil_{Z} \in$ $\left.\overline{\Lambda\left(\mathcal{L}\left(B_{Z}\right)\right)}\right\rceil_{Z}$. By (3i) we get that $\xi\left\lceil_{Z} \text { actually belongs to the set } \overline{\Lambda(\mathcal{L}(S))}\right\rceil_{Z}$. Pick $x^{*} \in \overline{\Lambda(\mathcal{L}(S))}$ so that $\xi\left\lceil_{z}=x^{*}\left\lceil z\right.\right.$. For every $i \in \mathbb{N}$ find $x_{i}^{*} \in \Lambda(\mathcal{L}(S))$ so that $\left\|x^{*}-x_{i}^{*}\right\|<\frac{1}{i}$. A moment reflection reveals that for every $i \in \mathbb{N}$ there is $m_{i} \in \mathbb{N}$ so that $x_{i}^{*} \in \Lambda\left(\mathcal{L}\left(S_{m_{i}-1}\right)\right)$. Then, by (4),

$$
\begin{aligned}
\left\|\xi-x_{i}^{*}\right\| & =\sup \left\{\langle\xi, s\rangle-\left\langle x_{i}^{*}, s\right\rangle: s \in S_{m_{i}}\right\} \\
& \left.\left.\left.\leq \| \xi\rceil_{Z}-x_{i}^{*}\right\rceil_{Z}\|=\| x^{*}\right\rceil_{Z}-x_{i}^{*}\right\rceil_{Z}\|\leq\| x^{*}-x_{i}^{*} \|<\frac{1}{i}
\end{aligned}
$$

for every $i \in \mathbb{N}$. Therefore $\xi \in \overline{\Lambda(\mathcal{L}(S))} \subset \overline{\Lambda\left(\mathcal{L}\left(B_{V}\right)\right)}$, and so, $v^{*}=\xi \uparrow_{V} \in$ $\overline{\Lambda\left(\mathcal{L}\left(B_{V}\right)\right)} \uparrow_{V}$.

Finally, consider any subspace $V$ of $X$. Choosing a dense subset $M$ of $B_{V}$, with $\# M=\operatorname{dens} V$, we have by (3)

$$
\begin{aligned}
\operatorname{dens} V & \leq \operatorname{dens} V^{*} \leq \operatorname{dens} \overline{\Lambda\left(\mathcal{L}\left(B_{V}\right)\right)}\left\lceil_{V} \leq \operatorname{dens} \overline{\Lambda\left(\mathcal{L}\left(B_{V}\right)\right)}=\operatorname{dens} \overline{\operatorname{sp} \Lambda\left(\mathcal{L}\left(B_{V}\right)\right)}\right. \\
& =\operatorname{dens} \overline{\Lambda(\mathcal{L}(M))} \leq \# \Lambda(\mathcal{L}(M))=\# M=\operatorname{dens} V .
\end{aligned}
$$

Remark 2. Proposition 1 is crucial for dropping S. Simons' lemma from the original construction of a projectional resolution of the identity in duals to Asplund spaces; see [FG] modulo [F1]. Indeed, in [FG], instead of the mapping $\partial: \mathcal{C}\left(B_{X^{*}}\right) \longrightarrow 2^{B_{X^{*}}}$, there is considered the (so called duality) mapping $X \ni x \longmapsto\left\{x^{*} \in S_{X^{*}}:\left\langle x^{*}, x\right\rangle=\|x\|\right\}=: J(x)$. This $J: X \longrightarrow 2^{S_{X^{*}}}$ is also norm-to-weak* upper semicontinuous and weak* compact valued. Hence, the Jayne-Rogers theorem [F, Theorem 8.1.2] yields norm-to-norm continuous mappings $D_{1}, D_{2}, \ldots$ (now) from $X$ into $X^{*}$ such that for every $x \in X$ the limit $\lim _{n \rightarrow \infty} D_{n}(x)=: D_{0}(x)$ exists in the norm topology of $X^{*}$ and moreover $\left\langle D_{0}(x), x\right\rangle=\|x\|$. Yet, there is no obvious guarantee that the set $D_{0}(X)$ is equal to, or at least norm-dense in the unit sphere $S_{X^{*}}$. Here, S. Simons' lemma enters the argument and remedies the situation by showing that $D_{0}(X)$ is linearly dense in all of $X^{*}$.

Proposition 3. Let $(X,\|\cdot\|)$ be a non-separable Asplund space and let $Z \subset X$ be an infinite-dimensional subspace with dens $Z<\operatorname{dens} X$. Then there exists an overspace $Z \subset V \subset X$, with dens $V=\operatorname{dens} Z$, such that the restriction mapping $\overline{\operatorname{sp} \Lambda\left(\mathcal{L}\left(B_{V}\right)\right)} \ni x^{*} \longmapsto x^{*} \Gamma_{V}=: R\left(x^{*}\right) \in V^{*}$ is a (surjective) isometry.

Proof. Put $\aleph:=$ dens $Z$. By induction, we shall construct sets $S_{0} \subset S_{1} \subset S_{2} \subset$ $\cdots \subset B_{X}$, all of cardinality $\aleph$, as follows. Let $S_{0}$ be a dense subset of $B_{Z}$, with 
$\# S_{0}=\aleph$. Let $m \in \mathbb{N}$ be fixed and assume that we have already found $S_{m-1}$. We already know that $\# \mathcal{L}\left(S_{m-1}\right)=\aleph$. Hence, by the norm-to-norm continuity of the mappings $\lambda_{j}$ 's, we have that $\overline{\operatorname{sp} \Lambda\left(\mathcal{L}\left(S_{m-1}\right)\right)}$ is a subspace of $X^{*}$, with density $\aleph ;$ let $M$ be a dense set in it, with $\# M=\aleph$. Find a set $S_{m-1} \subset S_{m} \subset B_{X}$, with $\# S_{m}=\aleph$, so big that $\operatorname{sp} S_{m-1} \cap B_{X} \subset \overline{S_{m}}$ and that $\|\xi\|=\sup \left\langle\xi, S_{m}\right\rangle$ for every $\xi \in M$. Then, of course, $\left\|x^{*}\right\|=\sup \left\langle x^{*}, S_{m}\right\rangle$ for every $x^{*} \in \operatorname{sp} \Lambda\left(\mathcal{L}\left(S_{m-1}\right)\right)$. This finishes the induction (step).

Having constructed the $S_{m}$ for every $m \in \mathbb{N}$, put $S:=S_{1} \cup S_{2} \cup \cdots$ and $V:=\overline{\operatorname{sp} S}$; then $\# S=\aleph$ and $V$ is a subspace of $X$, with density $\aleph$. We shall show that this $V$, together with the corresponding $R$, serve for the conclusion of our proposition. Consider any $x^{*} \in \overline{\operatorname{sp} \Lambda\left(\mathcal{L}\left(B_{V}\right)\right)}$ and let $\varepsilon>0$ be arbitrary. It is easy to verify that $\bar{S}=B_{V}$. By (3i) $), \overline{\Lambda\left(\mathcal{L}\left(B_{V}\right)\right)}=\overline{\Lambda(\mathcal{L}(S))}$, and hence $\overline{\operatorname{sp} \Lambda\left(\mathcal{L}\left(B_{V}\right)\right)}=$ $\overline{\operatorname{sp} \Lambda(\mathcal{L}(S))}$. Thus $x^{*}$ belongs to $\overline{\operatorname{sp} \Lambda(\mathcal{L}(S))}$. Find then $\xi \in \operatorname{sp} \Lambda(\mathcal{L}(S))$ so that $\left\|x^{*}-\xi\right\|<\varepsilon$. We remark that $\xi$ belongs even to $\operatorname{sp} \Lambda\left(\mathcal{L}\left(S_{m-1}\right)\right)$ for some big $m \in \mathbb{N}$. Then

$$
\begin{aligned}
\left\|x^{*}\right\|-\varepsilon & <\|\xi\|=\sup \left\langle\xi, S_{m}\right\rangle \leq \sup \left\langle\xi, B_{V}\right\rangle \\
& =\|R(\xi)\|<\left\|R\left(x^{*}\right)\right\|+\varepsilon \leq\left\|x^{*}\right\|+\varepsilon .
\end{aligned}
$$

Thus $\left\|x^{*}\right\|=\left\|R\left(x^{*}\right)\right\|$. We proved that $R$ is an isometry. That $R$ is surjective follows immediately from Proposition 1 .

Proposition 4. Let $V$ be a subspace of an Asplund space $(X,\|\cdot\|)$ such that the restriction mapping $\left.\overline{\operatorname{sp} \Lambda\left(\mathcal{L}\left(B_{V}\right)\right)} \ni x^{*} \longmapsto x^{*}\right|_{V}=: R\left(x^{*}\right) \in V^{*}$ is a (surjective) isometry. Then the mapping $X^{*} \ni x^{*} \longmapsto R^{-1}\left(x^{*} \uparrow_{V}\right)=: P\left(x^{*}\right)$ is a linear norm-1 projection, $P\left(X^{*}\right)=\overline{\operatorname{sp} \Lambda\left(\mathcal{L}\left(B_{V}\right)\right)}$, dens $P\left(X^{*}\right)=\operatorname{dens} V$, and $\bar{V}^{w^{*}}=$ $P^{*}\left(X^{* *}\right)$.

Proof. The first three statements concerning $P$ immediately follow from the definition of it. The "density" statement is contained in Proposition 1. It remains to prove the last equality. That $V \subset P^{*}\left(X^{* *}\right)$ follows from the definition of $P$; hence $\bar{V}^{w^{*}} \subset P^{*}\left(X^{* *}\right)$. Assume there exists $x^{* *} \in P^{*}\left(X^{* *}\right) \backslash \bar{V}^{w^{*}}$. The Hahn-Banach separation theorem yields an $x^{*} \in X^{*}$ such that $\left\langle x^{* *}, x^{*}\right\rangle \neq 0$ and $\left.x^{*}\right|_{V} \equiv 0$. But

$$
\left\langle x^{* *}, x^{*}\right\rangle=\left\langle P^{*}\left(x^{* *}\right), x^{*}\right\rangle=\left\langle x^{* *}, P\left(x^{*}\right)\right\rangle=\left\langle x^{* *}, R^{-1}\left(\left.x^{*}\right|_{V}\right)\right\rangle=0 ;
$$

a contradiction.

Proposition 5. Let $V_{1}, V_{2}$ be two subspaces of an Asplund space $(X,\|\cdot\|)$ such that $V_{1} \subset V_{2}$ and that the restriction mappings $\overline{\operatorname{sp} \Lambda\left(\mathcal{L}\left(B_{V_{i}}\right)\right)} \ni x^{*} \longmapsto x^{*} \Gamma_{V_{i}}=$ : $R_{i}\left(x^{*}\right) \in V_{i}^{*}, i=1,2$, are (surjective) isometries. Define $P_{i}: X^{*} \rightarrow X^{*}$ by $P_{i}\left(x^{*}\right)=R_{i}^{-1}\left(x^{*} \uparrow_{i}\right), x^{*} \in X^{*}, i=1,2$. Then $P_{1} \circ P_{2}=P_{1}\left(=P_{2} \circ P_{1}\right)$, and $\left(P_{2}-P_{1}\right)\left(X^{*}\right)$ is isometrical with $\left(V_{2} / V_{1}\right)^{*}$. 
Proof. (i) From the definition of $P_{i}^{\prime}$ 's we have immediately that $P_{2} \circ P_{1}=P_{1}$. Now, consider any $x^{*} \in X^{*}$ and any $x^{* *} \in X^{* *}$. Since $\bar{V}_{1}^{w^{*}}=P_{1}^{*}\left(X^{* *}\right)$ by Proposition 4, there is a net $\left(v_{\tau}\right)_{\tau \in T}$ in $V_{1}$ which weak ${ }^{*}$ converges to $P_{1}^{*}\left(x^{* *}\right)$. Then, using the definition of $R_{2}$ and the inclusion $V_{1} \subset V_{2}$, we get

$$
\begin{aligned}
\left\langle P_{2}^{*} \circ P_{1}^{*}\left(x^{* *}\right), x^{*}\right\rangle & =\left\langle P_{1}^{*}\left(x^{* *}\right), P_{2}\left(x^{*}\right)\right\rangle=\lim _{\tau \in T}\left\langle P_{2}\left(x^{*}\right), v_{\tau}\right\rangle \\
& =\lim _{\tau \in T}\left\langle R_{2}^{-1}\left(x^{*}\left\lceil V_{2}\right), v_{\tau}\right\rangle=\lim _{\tau \in T}\left\langle x^{*}, v_{\tau}\right\rangle=\left\langle P_{1}^{*}\left(x^{* *}\right), x^{*}\right\rangle\right.
\end{aligned}
$$

and so $P_{2}^{*} \circ P_{1}^{*}=P_{1}^{*}$, that is, $P_{1} \circ P_{2}=P_{1}$. The "isometrical" statement can be shown as in the proof of [F, Proposition 6.1.9(iv)].

Now we are armed to construct a projectional resolution of the identity on the dual to every Asplund space. But we rather prefer to present a bit stronger/richer statement.

Theorem 6. Let $(X,\|\cdot\|)$ be a non-separable Asplund space. Then there exist a family $\mathcal{V}$ of subspaces of $X$ and a family $\left\{Y_{V}: V \in \mathcal{V}\right\}$ of subspaces of $X^{*}$ such that

(i) $\bigcup\{V: V \in \mathcal{V}$ and $\operatorname{dens} V=\aleph\}=X$ and $\bigcup\left\{Y_{V}: V \in \mathcal{V}\right.$ and $\left.\operatorname{dens} V=\aleph\right\}$ $=X^{*}$ for every infinite cardinal $\aleph<\operatorname{dens} X$;

(ii) if $V_{1}, V_{2} \in \mathcal{V}$, there is $V \in \mathcal{V}$ such that $V \supset V_{1} \cup V_{2}$ and $\operatorname{dens} V=$ $\max \left\{\operatorname{dens} V_{1}\right.$, dens $\left.V_{2}\right\}$.

(iii) for every $V \in \mathcal{V}$ the assignment $Y_{V} \ni x^{*} \longmapsto x^{*} \uparrow_{V}=: R_{V}\left(x^{*}\right) \in V^{*}$ is a surjective isometry, and hence the mapping $X^{*} \ni x^{*} \longmapsto R_{V}^{-1}\left(x^{*} \uparrow_{V}\right)=$ : $P_{V}\left(x^{*}\right)$ is a norm-1 linear projection on $X^{*}$, with range $Y_{V}$, and dens $P_{V}\left(X^{*}\right)=$ dens $V$;

(iv) $\bar{V}^{w^{*}}=P_{V}^{*}\left(X^{* *}\right)$ for every $V \in \mathcal{V}$;

$(\mathrm{v}) \mathcal{V}$ is complete in the following sense: if $\gamma$ is a limit ordinal, and $\left\{V_{\alpha}\right.$ : $1 \leq \alpha<\gamma\}$ is an increasing long sequence of elements of $\mathcal{V}$, then $V:=$ $\overline{\bigcup_{1 \leq \alpha<\gamma} V_{\alpha}}$ belongs to $\mathcal{V}$ and $Y_{V}=\overline{\bigcup_{1 \leq \alpha<\gamma} Y_{V_{\alpha}}}$

(vi) if $V, U \in \mathcal{V}$ and $V \subset U$, then $Y_{V} \subset Y_{U}, P_{V} \circ P_{U}=P_{V}\left(=P_{U} \circ P_{V}\right)$, and $\left(P_{U}-P_{V}\right)\left(X^{*}\right)$ is isometrical with $(U / V)^{*}$.

Proof. For every subspace $V$ of $X$ we put $Y_{V}:=\overline{\operatorname{sp} \Lambda\left(\mathcal{L}\left(B_{V}\right)\right)}$ and we consider the assignment $\left.Y_{V} \ni x^{*} \longmapsto x^{*}\right|_{V}=: R_{V}\left(x^{*}\right) \in V^{*}$. Let $\mathcal{V}$ consist of all subspaces $V$ of $X$ such that $R_{V}: Y_{V} \longrightarrow V^{*}$ is a surjective isometry.

(i) and (ii) are guaranteed by Propositions 3 and 1 .

(iii) follows from the definition of $\mathcal{V}$ via Propositions 1 and 4.

(iv) follows from Proposition 4 . 
(v) Assume the premise here holds. (3) and some elementary reasoning yields

$$
\begin{aligned}
\Lambda\left(\mathcal{L}\left(B_{V}\right)\right) & \subset \overline{\Lambda\left(\mathcal{L}\left(B_{V}\right)\right)}=\overline{\Lambda\left(\mathcal{L}\left(\overline{\bigcup_{\alpha<\gamma} B_{V_{\alpha}}}\right)\right)}=\overline{\Lambda\left(\mathcal{L}\left(\bigcup_{\alpha<\gamma} B_{V_{\alpha}}\right)\right)} \\
& =\overline{\bigcup_{\alpha<\gamma} \Lambda\left(\mathcal{L}\left(B_{V_{\alpha}}\right)\right)} \subset \overline{\bigcup_{\alpha<\gamma} \overline{\operatorname{sp} \Lambda\left(\mathcal{L}\left(B_{V_{\alpha}}\right)\right)}}=\overline{\bigcup_{\alpha<\gamma} Y_{V_{\alpha}}}
\end{aligned}
$$

Hence

$$
Y_{V}=\overline{\operatorname{sp} \Lambda\left(\mathcal{L}\left(B_{V}\right)\right)}=\overline{\bigcup_{\alpha<\gamma} Y_{V_{\alpha}}}
$$

It remains to show that $V \in \mathcal{V}$, that is, that the mapping $R_{V}: Y_{V} \longrightarrow V^{*}$ is a surjective isometry. By Proposition 1 and (5), $R_{V}$ is surjective. Further, fix any $x^{*} \in Y_{V}$ and let $\varepsilon>0$ be arbitrary. Find $\xi \in \bigcup_{\alpha<\gamma} Y_{\alpha}$ such that $\left\|x^{*}-\xi\right\|<\varepsilon$. Then $\xi$ belongs to $Y_{\alpha}$ for some $\alpha<\gamma$. Now, as $V_{\alpha} \in \mathcal{V}$, we have

$$
\begin{aligned}
\left\|x^{*}\right\|-\varepsilon & <\|\xi\|=\left\|R_{V_{\alpha}}(\xi)\right\|=\| \xi\left\lceil_{V_{\alpha}}\|\leq\| \xi \uparrow_{V} \|\right. \\
& <\left\|x^{*} \uparrow_{V}\right\|+\varepsilon=\left\|R_{V}\left(x^{*}\right)\right\|+\varepsilon \leq\left\|x^{*}\right\|+\varepsilon .
\end{aligned}
$$

Therefore, $\left\|x^{*}\right\|=\left\|R_{V}\left(x^{*}\right)\right\|$ and the mapping $R_{V}$ is shown to be an isometry. Thus $V \in \mathcal{V}$.

(vi) immediately follows from Proposition 5 ,

Corollary 7. Let $(X,\|\cdot\|)$ be a non-separable Asplund space. Then $\left(X^{*},\|\cdot\|\right)$ admits

(i) [FG] a projectional resolution of the identity, and also

(ii) [K] a 1-projectional skeleton.

Proof. (i) Let $\mu$ be the first ordinal with $\# \mu=\operatorname{dens} X$. Find a dense subset $\left\{x_{\alpha}: \omega<\alpha<\mu, \alpha\right.$ is non-limit $\}$ in $X$. By (i) find a separable element $V_{\omega} \in \mathcal{V}$. Let $\gamma \in(\omega, \mu]$ be any ordinal an assume that we already found $V_{\alpha} \in \mathcal{V}$ for every $\omega \leq \alpha<\gamma$. Assume first that $\gamma$ is non-limit. By (i) find a $V \in \mathcal{V}$ such that $V \ni x_{\gamma}$ and dens $V=\operatorname{dens} V_{\gamma-1}$. By (ii) find a $V_{\gamma} \in \mathcal{V}$ such that $V_{\gamma} \supset V \cup V_{\gamma-1}$ and dens $V_{\gamma}=V_{\gamma-1}$. Second, assume that $\gamma$ is a limit ordinal. Put then $V_{\gamma}=\overline{\bigcup_{\omega<\alpha<\gamma} V_{\alpha}}$. By (v), we have that $V_{\gamma} \in \mathcal{V}$ and $Y_{V_{\gamma}}=\overline{\bigcup_{\omega \leq \alpha<\gamma} Y_{V_{\alpha}}}$. Now we can immediately verify that $\left\{P_{V_{\alpha}}: \omega \leq \alpha \leq \mu\right\}$ is a projectional resolution of the identity on $\left(X^{*},\|\cdot\|\right)$; see, e.g. [F, Definition 6.1.5].

(ii) We note that the relation " $\subset$ " on the family $\mathcal{V}$ from Theorem 6 is a directed partial order, which is moreover $\sigma$-complete. Thus, the subfamily $\left\{P_{V}: V \in\right.$ $\mathcal{V}$ and dens $\left.V=\aleph_{0}\right\}$ is a 1-projectional skeleton on $\left(X^{*},\|\cdot\|\right)$ in the sense of the definition in [KKL, pages 369, 370].

Remark 8. 1. A 1-projectional skeleton in the dual to an Asplund space was originally constructed by W. Kubiś $[\mathrm{K}]$. He started from the existence of the so called projectional generator in the dual to an Asplund space [F, Proposition 8.2.1] and then he proceeded using elementary submodels from logic. It should 
be noted that the proof of [F, Proposition 8.2.1] is based on S. Simons' lemma and on the Jayne-Rogers selection theorem [JR, Theorem 8].

2. Let $\|\cdot\|_{1},\|\cdot\|_{2}, \ldots$ be a sequence of equivalent norms on an Asplund space $X$. Let $\mathcal{V}_{1}, \mathcal{V}_{2}, \ldots$ be corresponding families found in Theorem 6 for these norms. It is not difficult to show that the family $\mathcal{V}:=\bigcap_{i=1}^{\infty} \mathcal{V}_{i}$ (is not only nonempty but that it even) satisfies all the conditions (i) - (vi) of Theorem 6; see the proof of [BM, Proposition 1.1]. Then Corollary 7 provides one projectional resolution of the identity and one 1-projectional skeleton on $X^{*}$ which can be related to any of the norms $\|\cdot\|_{1},\|\cdot\|_{2}, \ldots$

3. Theorem 6 can be proved also with help of tools from [FG] (where S. Simons' lemma was used). Indeed, let the mappings $D_{n}: X \rightarrow X^{*}, n \in \mathbb{N}$, be from Remark 2 (they come from the Jayne-Rogers theorem) and define $D: X \rightarrow 2^{X^{*}}$ by $D(x)=\left\{D_{1}(x), D_{2}(x), \ldots\right\}, x \in X$. For every subspace $W$ of $X$ put $Y_{W}:=$ $\overline{\operatorname{sp} D(W)}$ and consider the assignment $Y_{W} \ni x^{*} \longmapsto x^{*} \uparrow_{W}=: R_{W}\left(x^{*}\right) \in W^{*}$. Let $\mathcal{W}$ consist of all subspaces $W$ of $X$ such that $R_{W}: Y_{W} \longrightarrow W^{*}$ is a surjective isometry. From [FG, pages 145, 146], where S. Simons' lemma and other things were used, we know that

$$
\overline{\operatorname{sp} D(W) \Upsilon_{W}}=W^{*} \quad \text { for every subspace } \quad W \subset X .
$$

(This is a weakened analogue of our Proposition 1). By [F1, Lemma 1], for every $Z \subset X$, with dens $Z<$ dens $X$ there is a subspace $Z \subset W \subset X$, with dens $W=$ dens $Z$, such that $R_{W}$ is an isometry from $Y_{W}$ into $W^{*}$. (This is an analogue of our Proposition 3.) Using this, we easily get that $\overline{\operatorname{sp} D(W) \Upsilon_{W}}=\overline{\operatorname{sp} D(W)} \Upsilon_{W}$, and by (6), $R_{W}: Y_{W} \rightarrow W^{*}$ is a surjective isometry. Thus $W \in \mathcal{W}$. This way we get the properties (i) - (iv) and (vi) listed in Theorem 6. (v) can be proved as in Theorem 6, now from the norm-to-norm continuity of the $D_{n}$ 's.

4. We use an opportunity to fix some inaccuracy in the book $[\mathrm{F}]$. In the proof of [F, Proposition 8.2.1], on the page 152, the equality (2) should read as $\overline{\operatorname{sp} \Phi\left(B_{0}\right) \Upsilon_{Y}}=Y^{*}$ and the set $\Delta$ should be defined as $\overline{\Phi\left(B_{0}\right) \Upsilon_{Y}} \cap B_{Y^{*}}$.

Acknowledgment. We thank our colleague W. Kubiś for discussing the topic of this note.

\section{REFERENCES}

[BM] J.M. Borwein and W.B. Moors, Separable determination of integrability and minimality of the Clarke subdifferential mapping, Proc. Amer. Math. Soc., 128 (2000), 215-221.

[E] R. Engelking, General topology, Warszawa 1977.

[F] M.J. Fabian, Gâteaux differentiability of convex functions and topology - weak Asplund spaces, J. Wiley \& Sons, 1997.

[F1] M. Fabian, On projectional resolution of identity of the duals of certain Banach spaces, Bull. Aust. Math. Soc., 35 (1987), 363-371.

[FG] M. Fabian, G. Godefroy, The dual of every Asplund space admits a projectional resolution of the identity, Studia Math. 91 (1988), 141-151. 
PROJECTIONS IN DUALS TO ASPLUND SPACES MADE WITHOUT SIMONS' LEMMA 9

[JR] J.E. Jayne, C.A. Rogers, Borel selectors for upper semicontinuous maps, Acta Math. 155 (1985), 41-79.

[KKL] J. Kakol, W. Kubiś, M. López-Pellicer: Descriptive Topology in Selected Topics of Functional Analysis Developments in Mathematics, Vol. 24, Springer Science \& Business Media, New York, 2011.

[K] W. Kubiś, Banach spaces with projectional skeletons J. Math. Anal. Appl. 350 (2009) 758-776.

[Ph] R.R. Phelps, Convex functions, Monotone operators, and Differentiability, Lecture Notes in Math. No. 1364 , 2nd Edition, Berlin, Springer Verlag 1993.

[St] Ch. Stegall, Spaces of Lipschitz functions on Banach spaces, Functional Analysis Essen 1991, 265-278; Lect. Notes in Pure and Appl. Math. 150 New York, Dekker 1996.

Faculty of Mathematics and Physics, Sokolovská 83, 18675 Praha 8, Czech REPUBLIC

Mathematical Institute of Czech Academy of Sciences, Žitná 25, 11567 Praha 1, Czech Republic

E-mail address: cuthm5am@karlin.mff.cuni.cz

E-mail address: fabian@math.cas.cz 